\title{
Les refuges à Podocarpus sp. pl. de la forêt atlantique brésilienne: une analyse du passé pour mieux les protéger dans le futur
}

Marie-Pierre LEDRU ${ }^{1}$

Maria Luisa FerRaz Salatino ${ }^{2}$

${ }^{1}$ Ird, Ur 032 Great Ice

Apartado Postal 17.12.857

Quito

Équateur

2 Universidade de São Paul Departamento de Biociências

São Paulo SP

Brésil

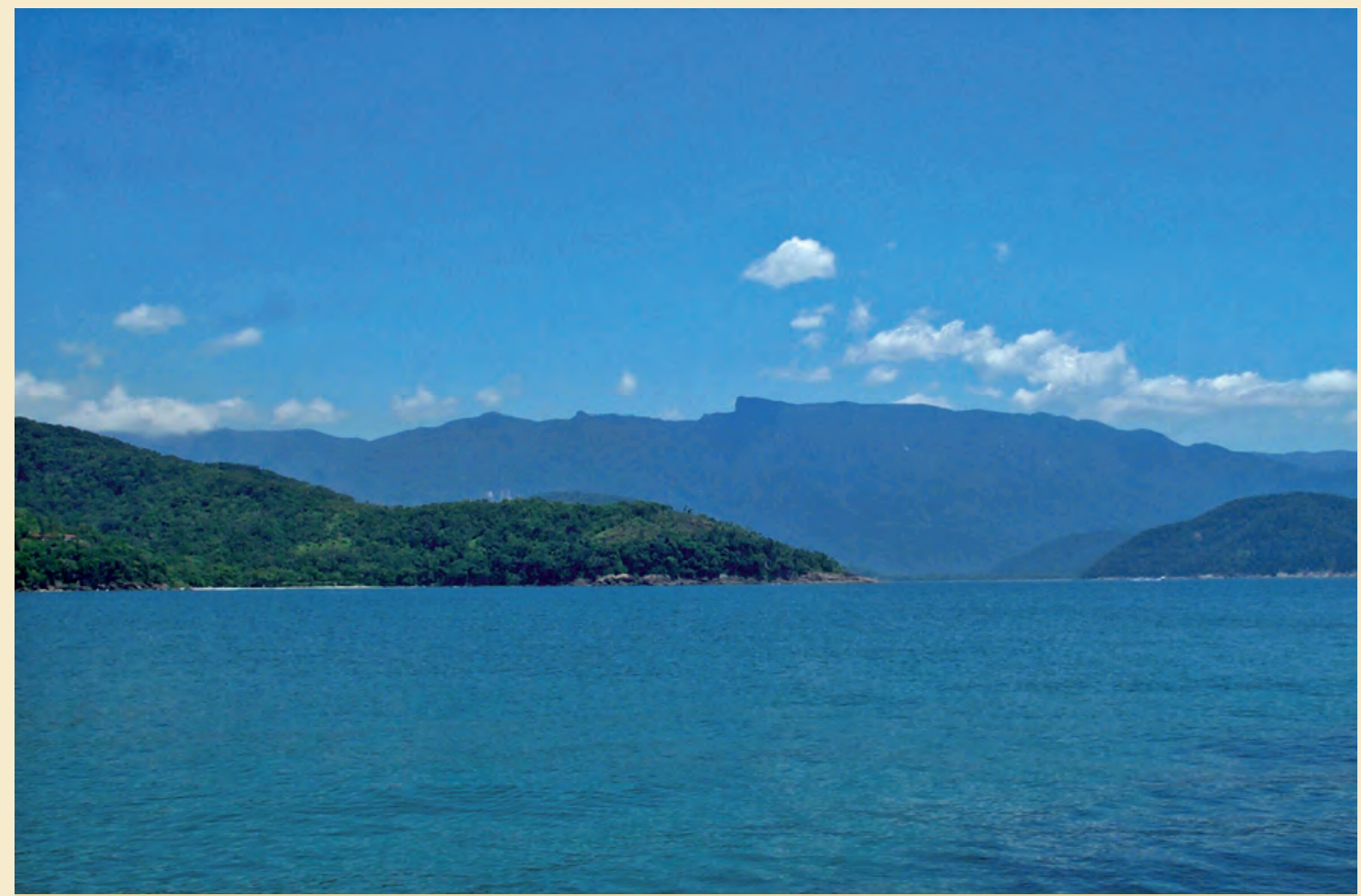

La forêt atlantique sur le littoral brésilien. Cette forêt est la mieux préservée aujourd'hui en raison des versants abrupts peu propices à l'agriculture. Elle s'étend sur le littoral entre $5^{\circ}$ et $30^{\circ} \mathrm{S}$ de latitude et comprend l'une ou l'autre espèce de Podocarpus selon sa localisation.

(C) M.-P. Ledru/Ird. 


\section{RÉSUMÉ}

\section{LES REFUGES À PODOCARPUS SP. PL. DE LA FORÊT ATLANTIQUE BRÉSILIENNE : UNE ANALYSE DU PASSÉ POUR MIEUX LES PROTÉGER DANS LE FUTUR}

La forêt pluviale atlantique est la deuxième pour la biodiversité après la forêt amazonienne. Située en grande partie dans les régions les plus agricoles du Brésil, elle a subi d'intenses déforestations au cours des deux derniers siècles. Aujourd'hui ne subsiste que $7 \%$ de sa couverture d'origine, pour la plupart des îlots de forêt ou une réserve écologique. Dans le but de définir l'expansion de la forêt atlantique pendant le quaternaire, nous avons inventorié les herbiers afin de localiser les populations des trois espèces endémiques de Podocarpus et extrait l'Adn des feuilles de 26 populations. Leur distribution actuelle est très étendue, entre l'équateur et $30^{\circ} \mathrm{S}$. La palynologie caractérise l'évolution temporelle des populations, la génétique permet de délimiter leur territoire d'expansion : entre $5^{\circ}$ et $15^{\circ} \mathrm{S}$, on retrouve les $P$. sellowii qui se sont développés il y a 16000 ans ; entre $15^{\circ}$ et $23^{\circ} \mathrm{S}$, des populations de $P$. lambertii ou sellowii se sont développées à plusieurs reprises depuis la dernière glaciation ; entre $23^{\circ}$ et $30^{\circ} \mathrm{S}$, P. lambertii est apparu en même temps que Araucaria, il y a 3000 ans. Le croisement de ces données permet de reconstituer les limites d'expansion pour des populations très dispersées aujourd'hui. Les refuges de la forêt atlantique que nous avons ainsi identifiés permettront à la forêt de survivre lorsque les conditions climatiques ne lui seront plus favorables et de se développer lorsque celles-ci redeviendront humides. Leur protection est donc cruciale pour l'avenir de la forêt atlantique et de sa biodiversité.

Mots-clés : forêt pluviale atlantique, Podocarpus, palynologie, changement climatique, refuge interglaciaire, Brésil.
ABSTRACT

\section{PODOCARPUS SP.PL. REFUGIA IN BRAZIL'S ATLANTIC FOREST: ANALYSING THE PAST TO IMPROVE FUTURE PROTECTION}

The Atlantic rainforest has the second highest biodiversity in Brazil. It has been shrinking rapidly in area as a result of intensive deforestation, and only $7 \%$ of the original cover now remains, as isolated patches or in ecological reserves. In order to obtain new information on the distribution of the Atlantic rainforest during the Quaternary, we examined herbarium data to locate relevant populations and extracted DNA from fresh leaves from 26 populations. The present-day distribution of endemic Podocarpus populations shows that they are widely dispersed across eastern Brazil, and that the expansion of Podocarpus recorded in single Amazonian pollen records may have originated from either western or eastern populations. Genetic analysis enabled us to determine the boundaries of their regional expansion: northern and central populations of $P$. sellowii appeared between $5^{\circ}$ and $15^{\circ} \mathrm{S}$ some 16,000 years ago; populations of P. lambertii or sellowii have appeared between $15^{\circ}$ and $23^{\circ} \mathrm{S}$ at different times since the last glaciation at least; and $P$. lambertii appeared between $23^{\circ}$ and $30^{\circ} \mathrm{S}$ during the recent expansion of Araucaria forests. The combination of botanical, pollen, and molecular analyses proved to be a rapid means of inferring distribution boundaries for sparse populations and their regional evolution within tropical ecosystems. Today the rainforest refugia we identified have become hotspots that are crucial to the survival of the Atlantic forest under unfavourable climatic conditions and, as such, offer the only possible opportunity for this type of forest to expand in the event of future climate change.

Keywords: Atlantic rainforest, Podocarpus, palynology, climate change, interglacial refugia, Brazil.

\section{RESUMEN}

\section{LOS REFUGIOS DE PODOCARPUS SP. PL. DE LA SELVA ATLÁNTICA BRASILEÑA: UN ANÁLISIS DEL PASADO PARA UNA MEJOR PROTECCIÓN EN EL FUTURO}

En Brasil, la selva pluvial atlántica ocupa, después de la selva amazónica, el segundo lugar en términos de biodiversidad, pero está mucho más amenazada que aquella. Situada en gran parte en las regiones más agrícolas del centro del país, ha sufrido una intensa deforestación a lo largo de los dos últimos siglos. Hoy en día subsiste apenas un $7 \%$ de su cobertura original, constituida en su mayor parte por islotes de selva en las vertientes de la cadena montañosa que bordea el litoral atlántico y, a veces, bajo la forma de reserva ecológica. Se realizó un estudio con el fin de caracterizar la distribución actual y pasada de las coníferas del género Podocarpus en Brasil, y de definir la extensión de la selva atlántica durante el Cuaternario. Se inventariaron los herbarios disponibles a fin de localizar las poblaciones de las tres especies endémicas pertenecientes al género y se extrajo el ADN de las hojas de 26 poblaciones. La palinología permitió caracterizar la evolución temporal de las poblaciones y la genética delimitar su territorio de expansión: entre $5^{\circ}$ y $15^{\circ} \mathrm{S}$ se encuentran los $P$. sellowii, que se desarrollaron hace aproximadamente 16000 años; entre $15^{\circ}$ y $23^{\circ} \mathrm{S}$ se desarrollaron poblaciones de $P$. lambertii o $P$. sellowii en varias ocasiones desde la última glaciación; y entre $23^{\circ}$ y $30^{\circ} \mathrm{S}$, P. lambertii apareció al mismo tiempo que las araucarias, hace aproximadamente 3000 años. Estos datos permiten reconstruir los límites de expansión de poblaciones arbóreas hoy muy dispersas. Los refugios forestales así identificados posibilitarán que la selva pluvial atlántica sobreviva en condiciones climáticas poco favorables y se desarrolle cuando tales condiciones se tornen más húmedas. La protección de esos refugios es pues crucial para el porvenir de la selva pluvial atlántica y su biodiversidad.

Palabras clave: selva pluvial atlántica, Podocarpus, palinología, cambio climático, refugio interglaciar, Brasil. 


\section{Introduction}

Les forêts tropicales sont doublement menacées aujourd'hui par l'homme et le réchauffement climatique et risquent d'avoir complètement disparu avant d'avoir été étudiées et comprises (MYERs et al., 2000). Cela est le cas de la forêt atlantique brésilienne (AubrévilLe, 1961). Afin de mieux comprendre la distribution actuelle et passée de cet écosystème, une étude récente (LEDRU et al., 2007) a été réalisée en ce qui concerne plus particulièrement les conifères endémiques de la forêt atlantique appartenant au genre Podocarpus. Ces conifères, reliques du Gondwana et caractéristiques de l'hémisphère sud, ont été abondamment utilisés par les paléoenvironnementalistes pour les reconstitutions de la variabilité climatique du quaternaire (LEDRU et al., 2001). Malgré cela, le déterminisme de leur répartition n'est pas encore bien compris. Trois espèces endémiques sont distinguées aujourd'hui au Brésil : Podocarpus lambertii Klotzsch, P. sellowii Klotzsch ex-Endl. et $P$. brasiliensis Laubenfels (LAUBENFELS, 1985). Ces trois espèces sont présentes dans la forêt atlantique (les deux premières) ou dans les cerrados (les deux dernières). Toutefois, $P$. sellowii et $P$. lambertii peuvent se trouver ensemble sur la même parcelle et $P$. sellowii se développe de $5^{\circ} \mathrm{N}$ jusqu'à $30^{\circ} \mathrm{S}$ de latitude, du niveau de la mer à 1800 m d'altitude. Toutes ces populations, extrêmement fragmentées, répondent à une même condition climatique nécessaire : un apport constant d'humidité qui peut être édaphique comme, par exemple, les populations de forêt-galerie, qui peut être atmosphérique, c'est le cas des populations des forêts de nuage, ou qui peut être climatique, c'est-àdire une distribution de la pluie sur toute l'année, au sud et sur le littoral du Brésil (Oliveira Filho, Fontes, 2000 ; Oliveira Filho et al., 1994).

Pour mener le travail de recherche, ont été, en premier lieu, consultés les botanistes spécialistes

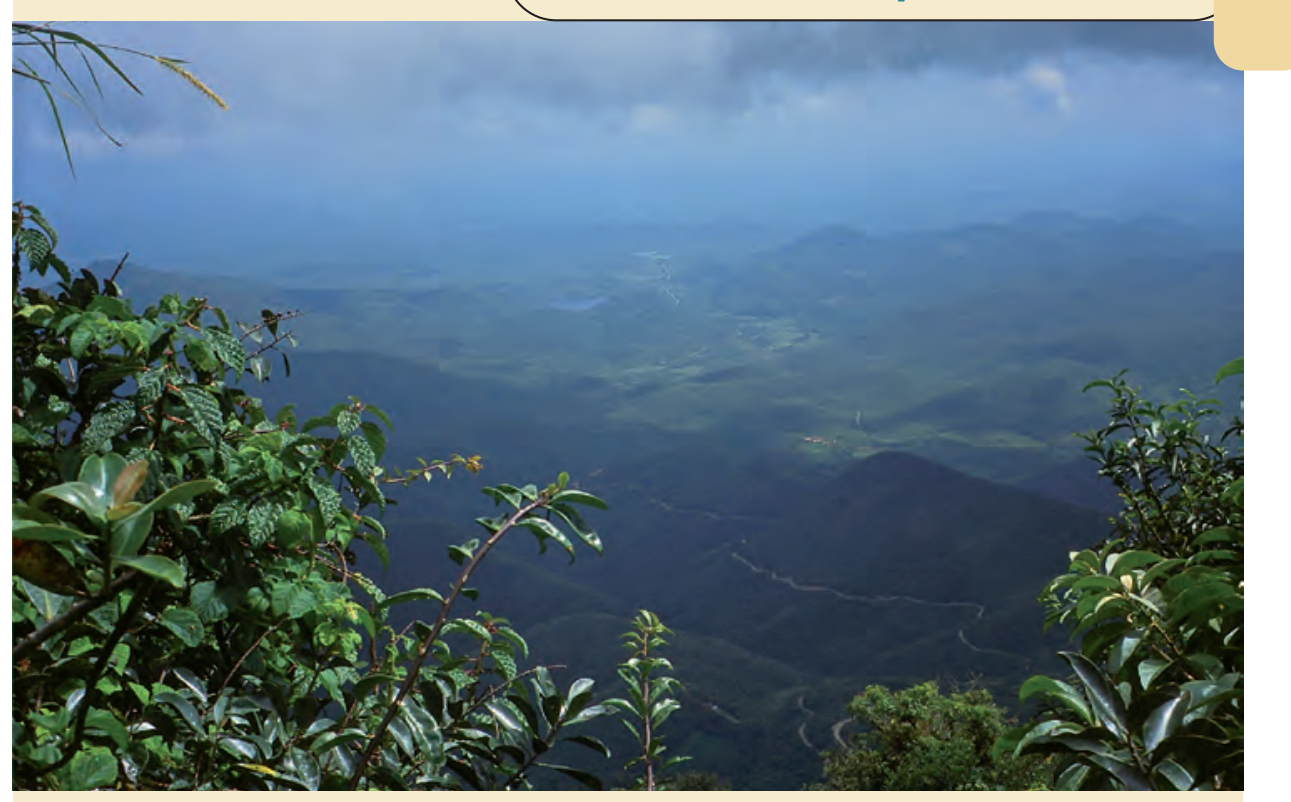

Refuge de forêt atlantique dans le Nordeste, près de Fortaleza. Les Podocarpus sellowii se développent dans cette région aride à la faveur de l'humidité apportée par les alizés et retenue sur les reliefs.

(c) M.-P. Ledru/Ird.

de ces forêts, puis les spécimens d’herbier ont été examinés. Les premiers résultats, très confus, ne permettaient pas de préciser les limites écologiques des distributions spatiales. Ainsi, en second lieu, il a été fait appel à la biologie moléculaire afin de retracer les éventuels liens de parenté entre les différentes mosaïques de forêt sur la totalité du territoire brésilien. Ces résultats, confrontés à ceux de la palynologie, ont permis de mieux comprendre le rôle que jouent ces populations de forêt humide dans la préservation de la forêt atlantique soumise à la succession des cycles glaciaire-interglaciaire du Quaternaire. La combinaison de ces trois disciplines, botanique, biologie moléculaire et palynologie, s'avère essentielle pour préciser le cadre d'évolution de la forêt tropicale, avec l'espoir qu'elle contribuera à sa préservation pour son évolution future. Cette présentation constitue une synthèse illustrée des résultats obtenus lors de l'étude (LEDRU et al., 2007), grâce au projet financé par la Fundação Amparo Pesquisa do Estado de São Paulo (FAPESP), le Mae-Brésil et un accord de coopération scientifique entre le CNPq (Brésil) et l'Ird (France).

\section{La forêt pluviale atlantique : une forêt disparue avant d'avoir révélé ses secrets}

La forêt pluviale atlantique est la deuxième pour la biodiversité après la forêt amazonienne et elle est beaucoup plus menacée que cette dernière (figure 1). En effet, son territoire se situant en grande partie dans les régions les plus agricoles du centre du Brésil, elle a subi d'intenses déforestations au cours des deux derniers siècles. Aujourd'hui, seuls $7 \%$ de sa couverture d'origine sont observés, constitués pour la plupart d'îlots de forêt accrochés aux versants de la chaîne de montagne bordant le littoral atlantique ou bien sous forme de réserve écologique. Pendant longtemps, il était considéré que les forêts du nord, du centre, du sud et de l'est du Brésil constituaient des domaines de forêt distincts. Des analyses statistiques réalisées sur des inventaires forestiers de populations disséminées sur tout le Brésil ont permis de revoir ces définitions (Oliveira Filho, Fontes, 2000). En 


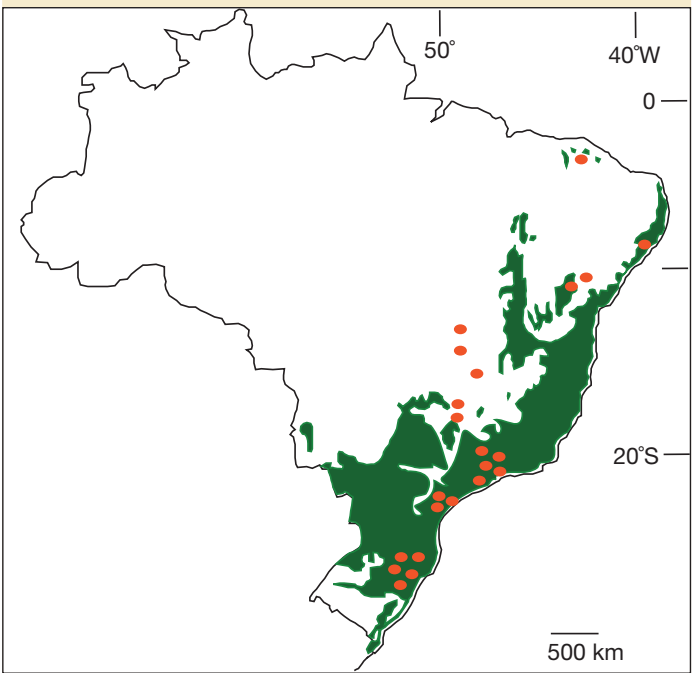

Figure 1.

Carte du Brésil montrant en vert la localisation de la forêt atlantique lato sensu et en rouge la répartition des populations de Podocarpus analysées pour cette étude.

effet, elles ont montré qu'il s'agissait d'un seul et unique domaine de forêt : la forêt atlantique lato sensu. Cette définition englobe les forêts ombrophiles denses, ombrophiles ouvertes, ombrophiles mixtes, semidécidues et décidues, la mangrove, la restinga, la végétation dunaire, les estuaires, les arbustes d'altitude (Joly et al., 1999).

Cette nouvelle définition a permis de réaliser un grand pas pour la préservation de la forêt atlantique : en évaluant son étendue originelle, il devient possible de comprendre l'ampleur de sa destruction. Or, aux destructions d'origine anthropique, s'ajoute depuis peu un autre facteur de disparition de cette forêt tropicale : le changement climatique. Mais, dans le passé, quel avait été son comportement face aux changements climatiques ayant affecté les basses latitudes sud-américaines? Afin de répondre à cette question, l'équipe de recherche a effectué une synthèse des résultats des analyses palynologiques ayant enregistré les grains de pollen de Podocarpus entre l'équateur et le tropique du Capricorne.

\section{Les évolutions climatiques quaternaires de la forêt atlantique}

Les grains de pollen de Podocarpus sont retrouvés dans trois domaines actuels de végétation : la forêt atlantique, la forêt amazonienne et le cerrado. À chaque fois, ils se trouvent associés à des taxons particuliers.

Dans le sud du Brésil, une augmentation des fréquences des grains de pollen des genres Podocarpus, Hedyosmum, Weinmannia et llex est interprétée en tant que développement de la forêt de nuage et, lorsque s'ajoute le genre Araucaria, expansion de la forêt d'araucaria. Le développement de la forêt de nuage y est relativement récent, et remonte à moins de 3000 ans. Le développement de la forêt d'araucaria est encore plus récent, puisqu'il date de moins de 1000 ans (LeDRU et al., 1994). Pour les périodes plus anciennes, un seul enregistrement est disponible à ce jour : il s'agit du site de Colônia, près de la ville de São Paulo. Podocarpus est retrouvé entre 27000 et 21000 ans, entre 60000 et 45000 ans et entre 100000 et 90000 ans avant le présent.
Dans le premier cas, il est associé aux espèces de la forêt de nuage humide et froide, dans les autres cas aux espèces de la forêt semi-décidue (LEDRU et al., 1996, 2006, 2007).

En Amazonie, l'association Podocarpus-Myrsine-Ilex, quelquefois accompagnée de HedyosmumWeinmannia, est enregistrée à deux reprises, au début de la déglaciation, environ 18000 ans avant le présent, et avant le maximum glaciaire, vers 25000 ans (LEDRU, 2002).

Dans le cerrado, Podocarpus est associé à llex et Myrsine, des taxons qui se retrouvent aussi dans les forêts des Andes. Une augmentation abrupte des fréquences de Podocarpus est enregistrée dans le nord et le centre du Brésil entre 16000 et 15000 ans avant le présent, suivie d'un développement de la forêt semi-décidue (LEDRU, 2002 ; LEDRU et al., 2006).

Les enregistrements palynologiques et notamment les fluctuations des fréquences des Podocarpus montrent que des changements importants ont eu lieu dans la répartition spatiale de la forêt atlantique depuis la dernière glaciation.

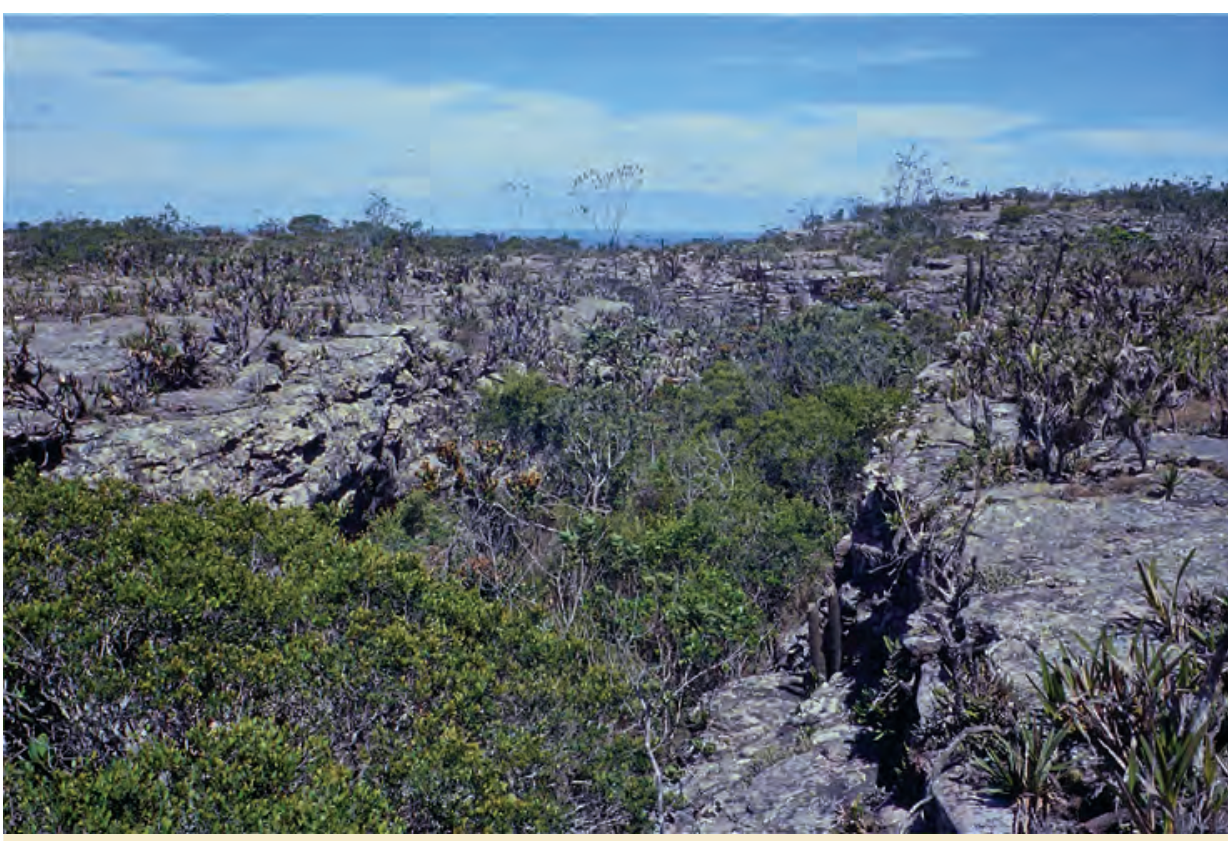

Refuge de Podocarpus dans l'État de Bahia. Les Podocarpus sellowii se développent à l'intérieur de " canaux » karstiques qui leur assurent une humidité permanente.

(c) M.-P. Ledru/Ird. 


\section{Quel lien entre les multiples fragments de forêt?}

Toutefois, les variations enregistrées par les grains de pollen sont très sporadiques et isolées : seuls sont disponibles une dizaine de sites palynologiques pour reconstituer les végétations sur la totalité du territoire brésilien. Des différences qu'il est parfois difficile d'expliquer sont observées d'un site à l'autre. La biologie moléculaire permet de reconstituer des parentés évolutives communes sur un plus vaste territoire et de relier entre elles les populations actuelles ainsi que les diagrammes palynologiques caractérisant une même évolution.
Les feuilles de 26 populations des trois espèces endémiques de Podocarpus réparties entre $30^{\circ} \mathrm{S}$ de latitude et l'équateur, entre 0 et $1800 \mathrm{~m}$ d'altitude, ont été récoltées afin de procéder à l'extraction de leur ADN (tableau I) à partir de protocoles et à l'aide de logiciels publiés (Vos et al., 1995 ; MACHALAKIS, EXCOFFIER, 1996 ; SWOFFoRD, 2002). Les résultats (figure 2) montrent tout d'abord que $P$. brasiliensis et $P$. sellowii ne font qu'une seule espèce, portant le nombre d'espèces endémiques à deux au lieu de trois. Ensuite, une nette séparation est observée entre les groupes à $P$. lambertii et ceux à $P$. sellowii. Les $P$. sellowii se séparent eux-mêmes en deux groupes, l'un situé entre les latitudes $5^{\circ}$ et $17^{\circ} \mathrm{S}$ et l'autre entre $17^{\circ}$ et $24^{\circ} \mathrm{S}$. Si la zonation principale de $P$. lambertii reste le sud du Brésil où il est associé à Araucaria angustifolia, il montre une plus ample distribution que $P$. sellowii puisqu'on le retrouve du nord au sud sous forme de mosaïques isolées ou de forêts.

Tableau 1.

Sites où les feuilles de Podocarpus destinées aux analyses de biologie moléculaire ont été récoltées avec leurs coordonnées géographiques. Les codes se rapportent aux numéros de la figure 2.

\begin{tabular}{|c|c|c|c|c|c|}
\hline Code & Herbier & Espèce & Altitude (m) & Latitude Sud & Longitude Ouest \\
\hline 1 & Ufce & P. lambertii & 880 & $14^{\circ} 14^{\prime}$ & $38^{\circ} 56^{\prime}$ \\
\hline 2 & Ufce & P. sellowii & 1100 & $8^{\circ} 18^{\prime}$ & $36^{\circ}$ \\
\hline 3 & Spf & P. cf. lambertii & 1400 & $13^{\circ} 32^{\prime}$ & $41^{\circ} 57^{\prime}$ \\
\hline 4 & Spf & P. sellowii & 1000 & $13^{\circ} 7^{\prime}$ & $41^{\circ} 7^{\prime}$ \\
\hline 5 & Spf & P. sellowii & 1000 & $14^{\circ} 13^{\prime}$ & $47^{\circ} 51^{\prime}$ \\
\hline 6 & Spf & P. brasiliensis & 750 & $15^{\circ} 86^{\prime}$ & $47^{\circ} 9^{\prime}$ \\
\hline 7 & Spf & P. sellowii & 1100 & $19^{\circ} 17^{\prime}$ & $43^{\circ} 35^{\prime}$ \\
\hline 8 & Spf & P. lambertii & 1100 & $19^{\circ} 17^{\prime}$ & $43^{\circ} 35^{\prime}$ \\
\hline 9 & Esal & P. sellowii & 1050 & $21^{\circ} 33^{\prime}$ & $44^{\circ} 38^{\prime}$ \\
\hline 10 & Spf & P. lambertii & 1200 & $22^{\circ} 75^{\prime}$ & $46^{\circ} 14^{\prime}$ \\
\hline 11 & Spf & P. sellowii & 950 & $20^{\circ} 11^{\prime}$ & $46^{\circ} 25^{\prime}$ \\
\hline 12 & Spf & P. lambertii & 1700 & $22^{\circ} 73^{\prime}$ & $45^{\circ} 59^{\prime}$ \\
\hline 13 & Pmsp & P. sellowii & 1100 & $23^{\circ} 59^{\prime}$ & $46^{\circ} 45^{\prime}$ \\
\hline 14 & Esal & P. sellowii & 0 & $25^{\circ} 01^{\prime}$ & $47^{\circ} 92^{\prime}$ \\
\hline 15 & Spf & P. sellowii & 26 & $25^{\circ} 8^{\prime}$ & $48^{\circ} 6^{\prime}$ \\
\hline 16 & Spf & P. lambertii & 875 & $25^{\circ} 43^{\prime}$ & $49^{\circ} 08^{\prime}$ \\
\hline 17 & Spf & P. sellowii & 1200 & $25^{\circ} 47^{\prime}$ & $48^{\circ} 33^{\prime}$ \\
\hline 18 & Paca & P. lambertii & 970 & $29^{\circ} 44^{\prime}$ & $50^{\circ} 58^{\prime}$ \\
\hline 19 & Paca & P. lambertii & 466 & $30^{\circ} 34^{\prime}$ & $52^{\circ} 44^{\prime}$ \\
\hline 20 & Spf & P. lambertii & 267 & $30^{\circ} 21^{\prime}$ & $53^{\circ} 37^{\prime}$ \\
\hline 21 & Spf & P. lambertii & 351 & $30^{\circ} 51^{\prime}$ & $53^{\circ} 09^{\prime}$ \\
\hline 22 & Spf & P. lambertii & 466 & $30^{\circ} 34^{\prime}$ & $52^{\circ} 44^{\prime}$ \\
\hline 23 & Spf & P. cf. montanus & 150 & $42^{\circ} 3^{\prime}$ & $72^{\circ} 15^{\prime}$ \\
\hline 24 & Spf & P. parlatorei & 2210 & $18^{\circ} 36^{\prime}$ & $64^{\circ} 01^{\prime}$ \\
\hline 25 & Spf & P. parlatorei & 2370 & $18^{\circ} 32^{\prime}$ & $64^{\circ} 02^{\prime}$ \\
\hline 26 & Spf & P. parlatorei & 1700 & $24^{\circ} 01^{\prime}$ & $65^{\circ} 23^{\prime}$ \\
\hline
\end{tabular}

Esal : Universidade de Lavras ; Paca : Porto Alegre Colegio Anchieta ; Pmsp : Prefeitura Municipal de São Paulo ; Ufce : Universidade Federal do Ceara. 


\section{Une histoire régionale différente selon la contrainte climatique}

Le croisement des données issues de la botanique, de la palynologie et de la biologie moléculaire a permis de délimiter un territoire évolutif commun pour chacun des groupes de populations. Trois régions principales sont ainsi distinguées, qui correspondent à trois histoires climatiques de la forêt atlantique. L'une est localisée entre $5^{\circ}$ et $15^{\circ} \mathrm{S}$, et montre que $P$. sellowii peuplait la forêt atlantique du nord et du centre du Brésil il y a environ 16000 ans. Aujourd'hui, il est représenté par quelques îlots sur les sommets des montagnes exposées aux alizés. La deuxième région, entre $15^{\circ}$ et $23^{\circ} \mathrm{S}$, est formée à la fois de $P$. sellowii et $P$. lambertii qui se sont développés tour à tour en fonction des conditions climatiques. Ainsi, la forêt d'araucaria était bien développée entre 60000 et 45000 ans, alors que c'est plutôt de la forêt semi-décidue qui s'étendait entre 27000 et 21000 ans et entre 100000 et 90000 ans avant le présent. Enfin, dans la troisième région, comprise entre $23^{\circ}$ et $30^{\circ}$ de latitude Sud, seul P. lambertii se propage depuis 3000 ans dans le sud du Brésil (LEDRU et al., 2007).

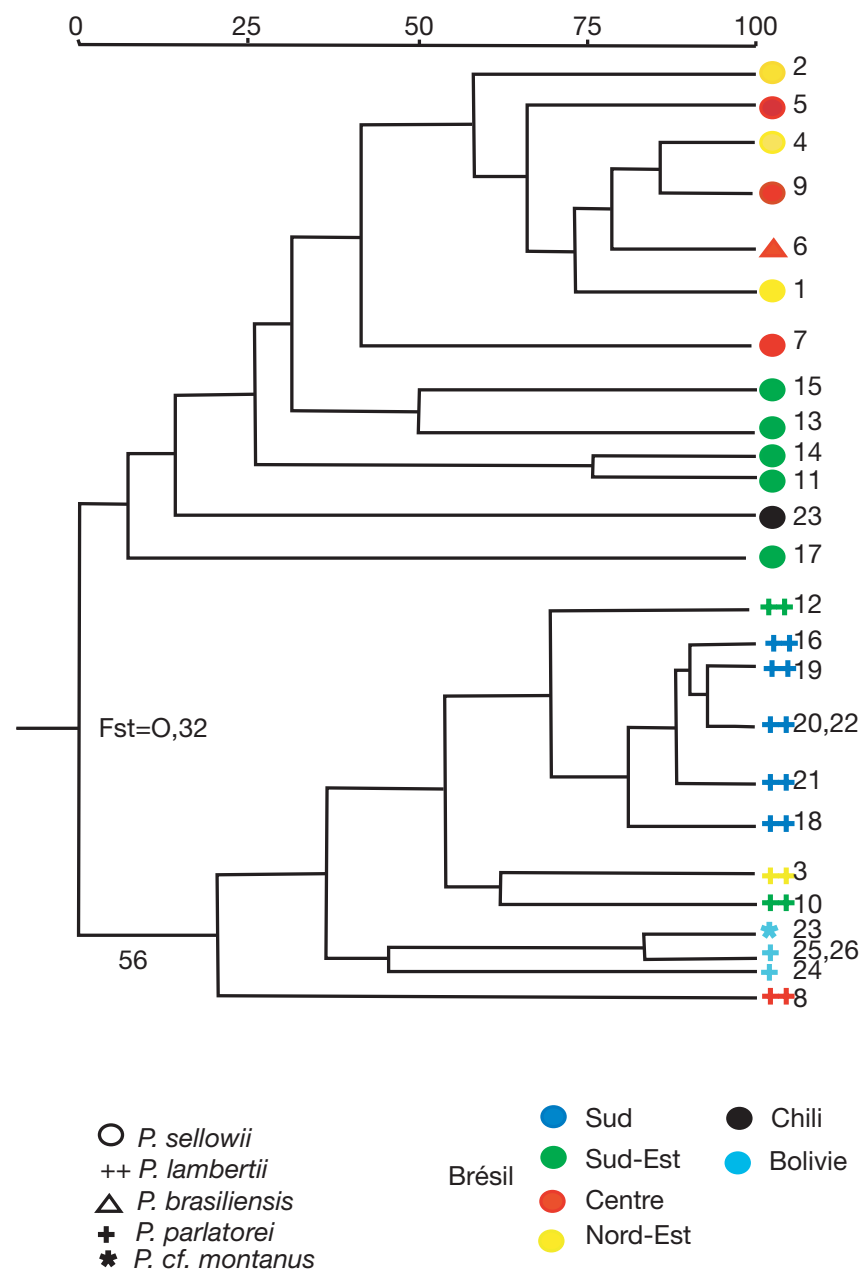

Figure 2.

Dendrogramme Upgma montrant la relation entre toutes les populations de Podocarpus analysées, basée sur la distance de Jacard. Les numéros correspondent à ceux du tableau I. Les couleurs indiquent les régions où les échantillons ont été prélevés.

\section{Homme, climat, quel futur pour la forêt atlantique?}

L'expansion passée de ces populations s'est faite très rapidement, à la faveur d'un changement climatique qui leur était favorable. Mais pour pouvoir répondre aussi rapidement à un changement climatique, les distances et l'éloignement des différentes populations doivent être réduits, comme il est possible de l'observer aujourd'hui dans le Nordeste ou dans les montagnes du centre du Brésil. Les résultats obtenus montrent clairement que la destruction de l'un ou l'autre de ces îlots de forêt augmentera la distance de rapprochement et pourra empêcher l'expansion de la forêt. Ces refuges actuels contribuent donc à la régénération de la forêt et au brassage de sa biodiversité lorsque se produira une expansion de la forêt atlantique. La préservation de ces refuges, aujourd'hui fortement menacés car localisés dans des régions économiquement pauvres, est essentielle à la survie de cette forêt face aux changements climatiques à venir. Depuis son arrivée sur le continent sudaméricain, il y a environ 12000 ans, l'homme a façonné le paysage afin de pouvoir y vivre et y survivre. À ces aménagements (construction de villages, pratique de la culture en terrasse, déboisements, développement de méthodes d'irrigation) se sont ajoutées les contraintes naturelles (variation du climat, éruption volcanique, glissement de terrain). Ces impacts sont-ils irréversibles pour l'écosystème ? Quel est le seuil à partir duquel cette action conjointe de l'homme et du climat devient irréversible et entraîne la création d'un nouvel environnement? Ces questions sont posées aujourd'hui alors que les décisions politiques de croissance économique ne sont pas en phase avec le problème de la transformation d'un paysage et de la dis- 
ponibilité des ressources naturelles sur lesquelles comptent les générations futures. Ces enjeux sont d'autant plus importants dans les pays en développement où la pauvreté des populations constitue une pression supplémentaire sur leurs ressources naturelles et où l'environnement est perçu comme un espace où se développent les activités humaines.

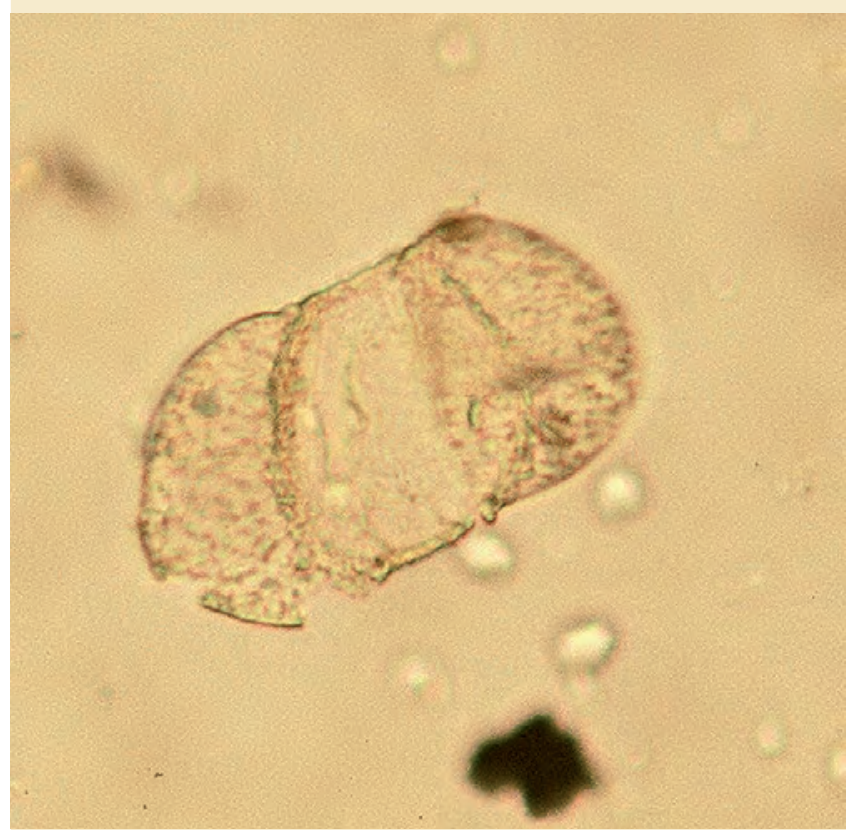

Grain de pollen de Podocarpus sp. grossi 1000 fois. Ce grain de pollen facile à identifier est un excellent marqueur pour reconstituer les expansions passées de la forêt atlantique. (C) M.-P. Ledru/Ird.

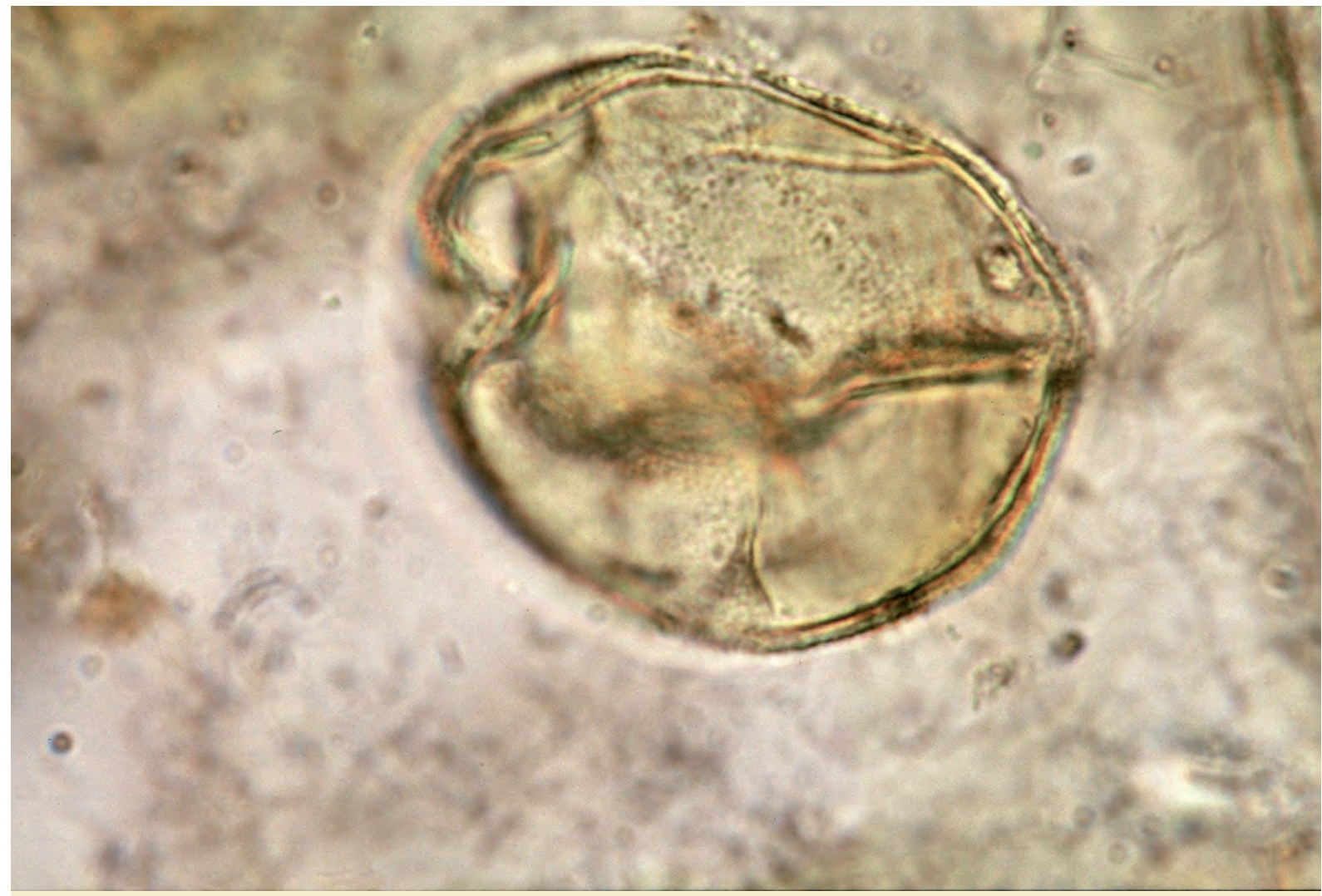

Grain de pollen d'Araucaria angustifolia grossi 1000 fois. A. angustifolia s'étend dans le sud du Brésil avec Podocarpus lambertii depuis 3000 ans. (c) M.-P. Ledru/Ird. 


\section{Références bibliographiques}

AUBRÉVILLE A., 1961. Étude écologique des principales formations végétales du Brésil et contribution à la connaissance des forêts de l'Amazonie brésilienne. Nogent-sur-Marne, France, Centre technique forestier tropical, 268 p.

JOLY C. A., AIDAR M. P. M., KLINK C. A., MCGRATH D. G., MOREIRA A. G., MOUTINHO P., NEPSTAD D. C., OLIVEIRA A. A., POTT A., RODAL M. J. N., SAMPAIO E. V. S. B., 1999. Evolution of the Brazilian phytogeography classification systems : implications for biodiversity conservation. Ciência e Cultura, $51: 331-348$.

LAUBENFELS D. J. (DE), 1985. A taxonomic revision of the genus Podocarpus. Blumea, 30 : 251-278.

LEDRU M.-P., BEHLING H., FOURNIER M., MARTIN L., SERVANT M., 1994. Localisation de la forêt d'Araucaria du Brésil au cours de l'Holocène. Implications paléoclimatiques. Comptes rendus de l'Académie des sciences de Paris, 317 : 517-521.

LEDRU M.-P., SOARES BRAGA P. I., SOUBIĖS F., FOURNIER M., MARTIN L., SUGUIO K., TURCQ B., 1996. The last 50,000 years in the Neotropics (southern Brazil) : evolution of vegetation and climate. Palaeogeography, Palaeoclimatology, Palaeoecology, 123 : 239-257.

LEDRU M.-P., CAMPELLO R. C., LANDIM DOMINGUEZ J. M., MARTIN L., MOURGUIART P., SIFEDDINE A., TURCQ B., 2001. Late-glacial cooling in Amazonia inferred from pollen at Lagoa do Caçó, northern Brazil. Quaternary Research, 55 : 47-56.

LEDRU M.-P., 2002. Late Quaternary history and evolution of the cerrados as revealed by palynological records. In : The cerrados of Brazil : Ecology and natural history of a neotropical savanna. Oliveira P. S., Marquis R. J. (éd.). New York, États-Unis, Columbia University Press, p. 33-52.
LEDRU M.-P., CECCANTINI G. T., PESSENDA L. C. R., LOPEZ J. A., GOUVEIA S. E. M., RIBEIRO A. S., 2006. Millennial-scale vegetation and climatic changes in a northern cerrado since the last glacial maximum. Quaternary Science Reviews, 25 : 1110-1126.

LEDRU M.-P., SALATINO M. L. F., CECCANTINI G. T., SALATINO A., PINHEIRO F., PINTAUD J.-C., 2007. Regional assessment of the impact of climatic change on the distribution of a tropical conifer in the lowlands South America. Diversity and Distributions, $13: 761-771$.

LEDRU M.-P., MOURGUIART P., RICCOMINI C., 2008. Related changes in biodiversity, insolation and climate in the Atlantic rainforest since the last Interglacial. Palaeogeography, Palaeoclimatology, Palaeoecology, on line.

MACHALAKIS Y., EXCOFFIER L., 1996. A genetic estimation of population subdivision using distances between alleles with special reference for microsatellite loci. Genetics, 142 : 1061-1064.

MYERS N., MITTERMEIER R. A., MITTERMEIER C. G., FONSECA G. A. B. D., KENT J., 2000. Biodiversity hotspots for conservation priorities. Nature, 403 : 853-858.

OLIVEIRA FILHO A. T., VILELA E. A., GAVILANES M. L., CARVALHO D. A., 1994. Comparison of woody flora and soils of six areas of montane semideciduous forest in southern Minas Gerais. Edinburgh Journal of Botany, 51 : 355-389.

OLIVEIRA FILHO A. T., FONTES M. A. L., 2000. Patterns of floristic differentiation among Atlantic forest in southeastern Brazil, and the influence of climate. Biotropica, 32 : 793-810.
OLIVEIRA FILHO A. T., CARVALHO D. A., FONTES M. A. L., VAN DEN BERG E., CURI N., CARVALHO W. A. C., 2004. Variações estruturais do compartimento arboreo de uma floresta semidecidua alto-montana na chapada das Perdizes, Carrancas, MG. Revista Brasileira Botânica, 27 : 291-309.

SWOFFORD D. L., 2002. Phylogenetic analysis using parsimony (* and other methods) version 4.0.b.10. Sunderland, MA, États-Unis, Sinauer Associates.

VOS P., HOGERS R., BLEEKER M., REIJANS M., VAN DE LEE T., HORNES M., FRIJTERS A., POT J., PELEMAN J., KUIPER M., ZABEAU M., 1995. AFLP : a new technique for DNA fingerprinting. Nucleic Acids Research, 23 : 4407-4414. 